Exercices Exercices de rhétorique

de rhétorique $\quad 10 \mid 2017$

Sur Térence

\title{
« Le Lion malade et le Renard » : un cas d'abduction chez La Fontaine
}

Franck Baron

\section{OpenEdition}

1 Journals

Édition électronique

URL : http://journals.openedition.org/rhetorique/577

DOI : 10.4000/rhetorique. 577

ISSN : 2270-6909

Éditeur

UGA Éditions/Université Grenoble Alpes

Édition imprimée

ISBN : 978-2-37747-029-7

\section{Référence électronique}

Franck Baron, « « Le Lion malade et le Renard » : un cas d'abduction chez La Fontaine », Exercices de rhétorique [En ligne], 10 | 2017, mis en ligne le 27 décembre 2017, consulté le 01 octobre 2020. URL: http://journals.openedition.org/rhetorique/577 ; DOI : https://doi.org/10.4000/rhetorique.577

Ce document a été généré automatiquement le 1 octobre 2020.

\section{(c) (1) () (2)}

Les contenus de la revue Exercices de rhétorique sont mis à disposition selon les termes de la Licence Creative Commons Attribution - Pas d'Utilisation Commerciale - Partage dans les Mêmes Conditions 4.0 International. 


\title{
« Le Lion malade et le Renard »: un cas d'abduction chez La Fontaine
}

\author{
Franck Baron
}

L'entendement semblable à l'œil, nous fait voir et comprendre toutes les autres choses, mais il ne s'aperçoit pas lui-même. C'est pourquoi il faut de l'art et des soins pour le placer à une certaine distance, et faire en sorte qu'il devienne l'objet de ses propres contemplations.

John Locke, Essai sur l'entendement humain, «Avant -propos », traduction de Pierre Coste

1 Dans Philosophies de la fable, Jean-Charles Darmon montrait chez La Fontaine la présence d'une réflexion épistémologique,

On s'interroge [...] sur les types d'expériences de pensée dont la littérature peut être porteuse, expérience de pensées qui, tout en entrant en dialogue avec celles de la science ou de la philosophie, n'obéissent pas aux mêmes procédures heuristiques ${ }^{1}$.

2 À sa suite, nous tenterons de questionner certains des mécanismes de la fable qui font accéder le «lecteur-modèle » au niveau réflexif. À quels types d'énoncés ou d'énonciations La Fontaine a-t-il recours? Comment ces énoncés permettent de tenir un discours épistémologique ? En substance, il s'agira de passer au crible de la « logique classique » la fiction fabuleuse afin de révéler les rapports qu'elles entretiennent. Il s'agira de mettre en question certains des concepts de la logique de l'âge classique, en émettant l'hypothèse que la fiction fabuleuse participe d'une pratique de la logique autant que d'un mode d'interrogation de ces concepts, à ne considérer que les débats sur les « animaux-machines ». 


\section{Approche pragmatique de la fable et de la logique}

3 Cette approche nécessite quelques rappels épistémologiques et méthodologiques, tant cette entreprise semble une véritable gageure aux yeux de nombreux chercheurs. Le premier de ces principes nous est fourni par le logicien Shahid Rahman :

Il existe une attitude aussi répandue que regrettable vis-à-vis de la logique, une attitude de déférence consistant à admettre ce que l'on présente comme ses lois immuables, dépourvue de tout sens critique. Elle repose sur l'hypothèse erronée que les principes fondamentaux de la logique sont (d'une manière ou d'une autre) des vérités inconditionnellement nécessaires, qui ne devraient pas être remises en question, que les notions de déduction et de démonstration ne doivent ou ne peuvent pas être soumis à un examen critique du point de vue philosophique ${ }^{2}$.

Il nous faudra également tenir compte de l'avertissement relayé par Olivier Caïra : "L'un des traits de la fiction mimétique est que nul n'est capable de dire quelle "portion de la réalité" est nécessaire à sa compréhension ${ }^{3}$ ». Cette citation ne se comprend qu'à la suite de celle de Jean Lassègue: "une formalisation [logique] n'a d'intérêt que si elle porte au contraire sur des portions limitées de la réalité dont on connaît déjà bien les propriétés ${ }^{4} »$. Autrement dit la logique ne peut pas restituer dans sa totalité une fiction, et elle ne nous permettra pas d'interroger directement la vérité de la fable, mais la petite portion centrée sur ces "procédures heuristiques ». Notre hypothèse de travail se veut également la poursuite des conclusions de Jean-Paul Sermain développées dans Métafictions :

Cette instabilité du sens des propriétés même de la métafiction est ce qui la rend difficile à cerner et à décrire : elle n'est pas dans ce qu'elle dit, mais dans ce qu'elle fait, par conséquent dans ce qu'elle fait faire, dans les parcours interprétatifs qu'elle prépare et dans les réactions qu'elle suscite ${ }^{5}$.

Comprendre d'abord à quoi servent les énoncés : ce qu'ils font, puis ce qu'ils font faire, avant que de pouvoir énoncer ce qu'ils disent, afin de montrer en quoi ils sont porteurs de sens. Il s'agira donc de partir du résultat pragmatique de la métafiction, de décrire les réactions qu'elle provoque sur le lecteur, pour retrouver une communauté « d'effets cognitifs » que rendrait sensible une logique pragmatique localisée à partir des énoncés épistémiques.

6 Ainsi à titre d'exemple, la construction logique de l'énoncé de la moralité des "Animaux malades de la Peste» semble n'admettre, dans la lettre, que deux cas possibles : «Selon que vous serez puissant ou misérable / Les jugements de cour vous rendront blanc ou noir ${ }^{6} »$. Or il doit pouvoir se présenter quatre cas de figure à envisager, quatre combinaisons, les cas où le " puissant » est soit « noir », soit « blanc », et les cas où le « misérable » est soit " noir », soit «blanc ». L'énoncé, dans le sens, ne dit pourtant pas qu'il soit possible qu'un «jugement de cour» puisse rendre noir un puissant, ou blanc un misérable. Ce qui paraît intuitivement faux. Il serait difficile de soutenir son caractère systématique; tous les jugements ne vous rendent pas noir ou blanc selon votre origine sociale! Ou alors il nous faut redéfinir la notion même de jugement ou de justice, ce qu'ici La Fontaine nous invite bien sûr à faire. Cette logique, bien que cohérente avec une certaine misanthropie lafontainienne ne paraît pas être valide. Mais ce qui importe dans cet exemple n'est pas de nier logiquement l'argument misanthropique de La Fontaine, ou la lourdeur sociale de l'étiquette, ni d'ergoter sur le fait que l'auteur aurait dû apporter un élément de logique modale dans sa démonstration pour atteindre un degré de véracité empiriquement acceptable. Et selon 
ce qui n'est dans ce cas qu'une hypothèse de lecture, de se demander si la forme, peutêtre volontairement invalide du raisonnement, ne participe pas pleinement au sens de la fable, à sa conclusion? Il s'agit de montrer que certains de ses énoncés peuvent être soumis à une analyse logique, pour surmonter la difficulté voire l'impossibilité théorique pour l'âge classique, de traiter ce genre d'énoncés par la syllogistique. Ce sont ces limites, ces écarts qui nous intéressent présentement. Tenter de comprendre cette distance, cette incommensurabilité, doit nous permettre de préciser comment écrivent ou pensent ces auteurs. Se contenter de dire dans l'exemple des «Animaux malades » que le raisonnement est invalide serait commettre une erreur grossière. $\mathrm{Ce}$ serait oublier le caractère perfectible et inachevé de cette logique (dans sa formulation par Port-Royal), autant que d'oublier la capacité d'invention des acteurs historiques, dans un champ qui relèverait $d u$ logique.

7 L'hypothèse que nous défendrons dans cet article est que l'une des formes d'énonciation à laquelle recourt La Fontaine est l'enthymème, syllogisme qui dévoilera le processus abductif lui-même. Ce syllogisme a la particularité de reposer sur l'utilisation de la «lumière naturelle » telle que préconisée par Port-Royal, et qui par certains égards peut se rapprocher d'une attitude pragmatique avant l'heure. Étudier « les actions de l'esprit » en mettant en avant une forme de naturalisation de la logique comme pour cet exemple tiré de L'Art de penser:

On a déjà dit que l'Enthymème était un syllogisme parfait dans l'esprit, mais imparfait dans l'expression; parce qu'on y supprime quelqu'une des propositions comme trop claire et trop connue, et comme étant facilement suppléée par l'esprit de ceux à qui on parle. Cette manière d'argument est si commune dans les discours et dans les écrits qu'il est rare, au contraire, que l'on y exprime toutes les propositions; parce qu'il y en a d'ordinaire une assez claire pour être supposée ; et que la nature de l'esprit humain est d'aimer mieux que l'on lui laisse quelque chose à suppléer, que non pas qu'on s'imagine qu'il ait besoin d'être instruit de tout ${ }^{7}$.

Attitude pragmatique que l'on retrouve aussi chez La Fontaine :

Que s'il m'est arrivé de le faire [se dispenser d'une moralité], ce n'a été que dans les endroits où elle n'a pu entrer avec grâce, et où il est aisé au lecteur de la suppléers.

Nous formulons donc l'hypothèse de l'utilisation rhétorique de l'enthymème comme une des procédures heuristiques de la fable.

\section{Usage de l'abduction chez La Fontaine}

10 Soit chez La Fontaine, un type d'énoncé susceptible de rendre compte de certains de ces " effets cognitifs» et pragmatiques à l'œuvre dans la fiction fabuleuse du XvII ${ }^{\mathrm{e}}$ siècle. «Effets cognitifs » qui se rapprocheraient d'une pratique plus ou moins consciente de l'enthymème et de l'abduction. Ils se déploient de manière assez visible dans la fable « Le Lion malade et le renard » reproduite in extenso :

De par le Roi des Animaux,

Qui dans son antre était malade,

Fut fait savoir à ses Vassaux

Que chaque espèce en ambassade

Envoyât gens le visiter :

Sous promesse de bien traiter

Les Députés, eux et leur suite,

Foi de Lion très bien écrite,

Bon passeport contre la dent ; 
Contre la griffe tout autant.

L'édit du Prince s'exécute :

De chaque espèce on lui députe.

Les Renards gardant la maison,

Un d'eux en dit cette raison:

Les pas empreints sur la poussière

Par ceux qui s'en vont faire au malade leur cour,

Tous, sans exception, regardent sa tanière ;

Pas un ne marque de retour.

Cela nous met en méfiance.

Que Sa Majesté nous dispense :

Grand merci de son passeport.

Je le crois bon ; mais dans cet antre

Je vois fort bien comme l'on entre,

Et ne vois pas comme on en sort ${ }^{9}$.

11 Ce qui nous intéresse dans cette fable n'est pas tant la portée politique de sa conclusion, que le raisonnement en jeu, pour construire/déconstruire cette fable. Nous allons ainsi tenter de retrouver la logique de la fable en la soumettant à la «logique classique» développée autour des grammairiens de Port-Royal. Il nous faudra, enfin, tenir compte de la spécificité de l'enthymème comme le souligne Jorge Juan Vega y Vega :

L'enthymème ne correspond pas aux caractéristiques générales du syllogisme canonique. En ce sens, l'enthymème est un syllogisme négatif. [...] on ne peut pas y appliquer automatiquement les règles de la syllogistique. Pour ce faire, il faut que l'enthymème prenne au préalable la forme d'un syllogisme, qu'il se positivise ; qu'il se dé-forme en quelque sorte ${ }^{10}$.

Ainsi, commençons l'analyse logique de la conclusion-moralité de la fable du «Lion malade et du renard» qui se caractérise précisément par son absence formelle de "moralité ", et qu'il sera "aisé au lecteur de la suppléer", selon les dires de La Fontaine.

Soit l'énoncé :

(1) «Je vois fort bien comme l'on entre, / Et ne vois pas comme on en sort. ${ }^{11}$ »

Si nous reprenons cet énoncé, nous pouvons le décomposer en formules atomiques suivantes :

- Soit «Je vois fort bien comme l'on entre» correspondant à $(p)$. Cette formule atomique pourrait tout aussi bien relever d'une logique modale voire épistémique caractérisée par la présence du "Je vois [que]», susceptible de correspondre de manière sémantique à Je crois que ou Je sais que. Posons, peut-être de manière un peu arbitraire que «Je vois fort bien comme l'on entre" peut être substitué et devenir compatible avec la logique classique par la proposition : on entre. Il semble que cette distinction était déjà connue et soulevée par Arnauld et Nicole. Sur les propositions modales :

comme si je dis : je soutiens que la terre est ronde; je soutiens n'est qu'une proposition incidente, [...] cependant il est visible qu'elle ne fait partie ni du sujet ni de l'attribut : car cela n'y change rien du tout, et ils seraient conçus entièrement de la même sorte si je disais simplement, la terre est ronde ${ }^{12}$.

- Soit la proposition retenue :

$(2)(p)=$ on entre, d'où nous pouvons retenir la négation suivante : non- $p,(\neg p)=$ on n'entre pas.

De la même manière nous traitons la formule atomique de «[je] ne vois pas comme on en sort » dont on " transfère » la négation sur le prédicat: Je vois qu'on ne sort pas, puis on « expurge » la modalité, pour obtenir la formule équivalente à on ne sort pas. 
- Soit la proposition retenue :

(3) non- $q,(\neg q)=$ on ne sort pas telle que $(q)=$ on sort.

Nous sommes désormais en mesure de traduire nos prémisses du langage naturel de la fiction en langage naturel restreint compatible avec la logique de Port-Royal, et ainsi obtenir son schéma d'argumentation, susceptible de restituer et d'évaluer la validité logique de la conclusion lafontainienne du "Lion malade et du renard». Dans une forme quasi syllogistique telle que Port-Royal aurait pu la formuler, on obtiendrait quelque chose comme :

On entre

Et on ne sort pas

Donc :

Nous voyons rapidement que Port-Royal et nous-mêmes sommes bien en peine de conclure quelque chose de ces deux seules prémisses. Il faut donc les reformuler.

- Soit «Je vois fort bien comme l'on entre, / Et ne vois pas comme on en sort» devenant dans le langage naturel restreint: Si on entre, alors on ne sort pas,

soit : (4) $p$ implique non- $q,(p \rightarrow \neg q)$.

Précisons qu'il aurait été tout à fait possible de traduire cette formule par: On entre et on ne sort pas, $\left(p^{\wedge} \neg q\right)$. Cependant, ceci n'aurait pas rendu avec autant de force le caractère explicatif que revête intuitivement cet énoncé. En logique propositionnelle, $\left(p^{\wedge} \neg q\right)$ est égal à $\left(\neg q^{\wedge} p\right)$, ce qui dans notre exemple serait faux (ou du moins plus étrange, car il romprait d'une certaine manière la temporalité de l'action et la causalité) et donnerait un nouvel énoncé à la fable; On ne sort pas et on entre. En paraphrasant un exemple proposé par Louis $\operatorname{Vax}^{13}$ : il se réveille et il descend du lit, n'est pas équivalent à il descend du lit et il se réveille. Enfin, nous pouvons logiquement utiliser l'implication en vertu de la règle de la négation des conditionnels : $(p \rightarrow q) \equiv \neg\left(p^{\wedge} \neg q\right)$. Ce qui dans notre formule (4) donne $(p \rightarrow \neg q) \equiv \neg\left(p^{\wedge} q\right)$. Soit en langage naturel (4) Si on entre alors on ne sort pas, équivalant à il n'est pas vrai que "on entre et on sort ", puisque précisément on entre et on ne sort pas. Comme le rappelle également Bernard Walliser et al. :

Leur assimilation [des conditionnels contrefactuels] à des implications matérielles ne permet pas de rendre compte du fait que ces énoncés peuvent être vrais ou faux non en vertu de la seule logique, mais en fonction de la réalité empirique. Elle ne rend pas compte non plus de l'intuition attachée à la "relation causale" entre l'antécédent et le conséquent que ces énoncés tendent à exprimer ${ }^{14}$.

Il nous faut maintenant déterminer la valeur de vérité de cette proposition $(p \rightarrow \neg q)$. C'est-à-dire s'assurer si $(p)$ est vraie ou fausse et $(\neg q)$ également. De ceci dépend la validité de l'argumentation de La Fontaine :

Un argument est valide ssi (si, et seulement si) il n'est pas possible que sa conclusion soit fausse alors que ses prémisses sont vraies. En d'autres termes : si les prémisses d'un argument valide sont vraies, alors sa conclusion doit être vraie également ${ }^{15}$.

Dans l'économie de cette fable, il y a bien dans le texte des phrases dénotant les objets textuels que sont "le Lion", "le Renard", "la tanière ", etc. sans contradiction apparente et ainsi dire qu'il existe bien (dans l'économie du texte ou Monde Textuel, devenu notre monde de référence) «Les pas empreints sur la poussière ${ }^{16}$ » établissant la vérité de $(p)=$ on entre. Et si j'ose dire, qu'il existe bien une absence de pas, puisque «Pas un[e empreinte de pas] ne marque de retour ${ }^{17}$ », établissant la vérité de la proposition $(-q)$, on ne sort pas.

- Soit donc la valeur de vérité de $(4): \mathrm{v}(p)=1$ et $\mathrm{v}(-q)=1$. 
En nous référant à la table de vérité de la proposition $(p \rightarrow \neg q)$, l'on constate que si $p$ est vraie et $\neg q$ est vraie, alors $(p \rightarrow \neg q)$ est valide.

[la définition de la validité d'un argument] est indifférente au fait que les prémisses soient, en réalité, vraies ou fausses. [...] Ce qui est important, c'est que si on accepte les prémisses (si on croit à leur vérité), on doit aussi accepter la conclusion de l'argument ${ }^{18}$.

Ici, l'implication matérielle ne saurait se confondre avec la causalité. Ainsi les propositions on entre et on ne sort pas sont causalement indépendantes. On ne sort pas ne nous apprend rien sur le fait d'entrer, et on entre ne nous dit pas pourquoi on ne sort pas. Pourtant l'énoncé lafontainien " Je vois fort bien comme l'on entre, / Et ne vois pas comme on en sort", placé en position de "conclusion» dans la fable semble indiquer que l'énonciateur, lui, est en mesure d'expliquer ce qui vient de se dérouler sous les yeux du lecteur, et de lui fournir une explication qui viendrait éclairer le sens de la fable. Si la fable se conclut par un raisonnement valide: l'implication matérielle, qui n'a pas de pouvoir heuristique, l'implication n'est pas explication. Si le lecteur veut savoir le fin mot de l'histoire, il lui faut donc poursuivre le raisonnement esquissé en conclusion. D'ailleurs cette idée du syllogisme comme formulation "inutile» car incapable de permettre la découverte ou l'explication de phénomène nouveau était déjà fort répandue :

Un auteur de ce temps [probablement Descartes] a dit avec grande raison, que les règles de la Logique d'Aristote servaient seulement à prouver à un autre ce que l'on savait déjà ${ }^{19}$.

16 La logique de Port-Royal montre-t-elle par là ses propres limites opératoires, que la logique de la fable tenterait de suppléer? La Fontaine invite donc par ce moyen, autant logique que rhétorique à poursuivre l'investigation fabuleuse. C'est précisément ici que l'analyse logique et formelle cède le pas à l'analyse pragmatique, à la «lumière naturelle»: en nous demandant à quoi sert cette formulation, puisqu'elle semble heuristiquement inopérante, voire hors de la logique du XvII ${ }^{\mathrm{e}}$ siècle, bien que valide?

\section{Besoin d'une seconde formulation : l'explication}

17 En elle-même, cette conclusion n'a rien de mystérieux, et tout lecteur attentif de La Fontaine l'infère aisément. Elle prend la forme de la proposition suivante : Si on entre et qu'on ne sort pas, alors (c'est parce que) le Lion a mangé les Députés. Il nous faut souligner ici le caractère explicatif que prend la conclusion « voilée » de la fable, et que ne rendait pas cette formulation logique initiale de l'implication. Si cette formulation ne rend que grossièrement toute la subtilité sémantique et épistémique de l'argumentation lafontainienne, elle permet de mettre en lumière le jeu, l'espace qui sépare la logique de l'âge classique de la «logique fabuleuse ». Bien que très imparfaite, cette confrontation des énoncés fictionnels ne doit se comprendre que comme un point de départ pour l'investigation historique et philosophique. C'est cet espace, la mise à jour explicite du décalage entre ces logiques qui permettra, peut-être, d'orienter en partie l'interprétation "philosophique » de la fiction fabuleuse. À ce stade de l'analyse, nous avons à peine évoqué le sens du message lafontainien, qu'il soit politique ou philosophique. Ce qui nous intéresse ici et pour l'instant, n'est pas le quoi du discours, mais bien son comment logique.

Soit donc un type d'énoncé que nous avons localisé dans le discours fabuleux: l'implication matérielle ou contrefactuelle, devenue en quelque sorte la colonne 
vertébrale du récit. Car ce raisonnement caché et volontairement incomplet est tout autant celui de l'auteur qui conclut sa fable, que celui de l'un des personnages :

Les Renards gardant la maison,

Un d'eux en dit cette raison:

Les pas empreints sur la poussière

Par ceux qui s'en vont faire au malade leur cour,

Tous, sans exception, regardent sa tanière ;

Pas un ne marque de retour.

Cela nous met en méfiance ${ }^{20}$.

19 Cette séquence narrative fait suite à ce que nous pourrions appeler la scène d'introduction où il est exposé que « le Roi des Animaux », "malade », reçoit la visite dans son antre de chaque espèce avec l'assurance de ne pas être mangé, résumé succinct des vers 1 à 12 . Voyons comment le Renard s'y prend pour construire son raisonnement et nous «di[re] cette raison». La Fontaine s'appuie ici sur un raisonnement indiciaire: "Les pas empreints sur la poussière [...] / Tous, sans exception, regardent sa tanière ». L'indice est matérialisé par la trace, "Les pas empreints » des Députés venus rendre visite au Roi.

\section{Signes et indices}

L'analyse indiciaire, héritée des scolastiques, se trouve au XvII siècle mobilisée aussi bien par les Messieurs ${ }^{21}$ que Marin Cureau de la Chambre avec son Art de connaitre les hommes :

Il y a trois moyens que cet art peut employer pour arriver à la fin qu'il se propose, \& qu'il peut découvrir un effet caché par la cause qui lui est connue, ou une cause obscure par un effet manifeste, \& un effet manifeste, \& un effet inconnu par un autre qui est euident. Et ces moyens sont appelés signes, parce qu'ils marquent \& désignent les choses qui sont obscures ${ }^{22}$.

21 L'indice y devient également la marque d'une causalité : « Ainsi la cendre chaude cache le feu comme chose, \& le découvre comme signe ${ }^{23}$ ", ou chez La Fontaine : "À l'œuvre on connaît l'artisan ${ }^{24} »$. On ne saurait confondre dans cette fable l'indice «des pas empreints » à la simple métonymie des Députés. Et cet énoncé les pas mènent à l'antre, aucun ne marque de retour, énoncé lui-même construit à partir des indices (physiques) des traces de pas (des députés), signe nécessaire : tekmérion, devient ainsi une sorte de méta-indice : les pas mènent à l'antre, aucun ne marque de retour est l'indice qu'il se trame quelque chose dans la tanière du roi, en devenant un signe probable, semeion ${ }^{25}$. Ainsi "cette raison" du renard tend à produire un schéma explicatif que la formulation logique - l'implication matérielle - ne permettait pas d'atteindre. Il faut rapprocher cette « logique » de La Fontaine de celle relevée par Sylvie Catellin dans les contes du XVIII ${ }^{\mathrm{e}}$ siècle français :

Zadig s'inspire de la science de son temps pour lire dans le « livre de la nature »: il analyse la forme des sillons entre les traces de pattes laissées par la chienne; il mesure la distance entre les marques des fers du cheval; il met en relation la largeur de la route et la place des traces de poussière ou les feuilles tombées des arbres pour évaluer la longueur de la queue du cheval ${ }^{26}[\ldots]$.

Dans cet exemple tiré du Zadig de Voltaire, passage lui-même partiellement repris d'un conte publié par le Chevalier Mailly ${ }^{27}$, nous pouvons résumer cette méthode de la manière suivante: Zadig met en relation des informations (étant elles-mêmes des indices) qui entre elles n'ont pas de liens de causalité directe ou directement 
observable. Pourtant Zadig parviendra à reconstruire une histoire et à fournir une explication sur la disparition du cheval recherché par son propriétaire. L'explication dans la fable du «Lion malade et le Renard » semble bâtie sur le même modèle, par la mise en relation d'informations qui sont causalement indépendantes, telles que formulées dans la logique du premier ordre: $(p \rightarrow \neg q)$, aboutissant en $\left(p^{\wedge} \neg q\right) \rightarrow r$, tentative maladroite pour restituer une formulation de la causalité : l'abduction.

Les formes de connaissance indiciaire font largement appel à la pensée conjecturale, basée sur ce que l'on appelle communément l'intuition (le flair), et sur ce qui relève aussi des croyances des individus, celles-ci étant réajustées en permanence par les opérations qu'ils font subir, parfois inconsciemment, aux informations qu'ils sélectionnent. Chez Aristote, l'intuition peut dans certains cas guider la dialectique et elle est appelée alors apagogè. Peirce renvoie lui-même à la notion d'apagogè pour introduire l'abduction ${ }^{28}$.

\section{Vers l'abduction : le doute}

Dès lors si le Renard dans son raisonnement parvient à relier de manière causale deux événements non causalement liés, comment s'y prend-il ? Il nous faut ici revenir sur l'aspect épistémique de la constatation lafontainienne : «Je vois fort bien comme l'on entre, / Et ne vois pas comme on en sort ». Pour Catellin, la sérendipité, cet art de faire des découvertes par hasard ${ }^{29}$ a pour caractéristique principale "la relation soudaine entrevue entre deux idées ». La "soudaineté » de ce phénomène tient pour partie en une disposition psychologique :

Le moteur de la recherche, notait Peirce dès 1877, est l'irritation produite par le doute, car le doute a pour effet de déstabiliser un état de croyance $^{30}$.

Cette disposition est dénotée dans la fable par ce commentaire du Renard à la suite de ces propres constatations: "Cela nous met en méfiance ${ }^{31}$ ». Il devient possible de traduire la formule Je vois / Je ne vois pas des vers 23-24, comme la marque chez l'énonciateur La Fontaine d'une forme, ici feinte, d'incompréhension, équivalente à la formule: Je vois comme l'on entre et ne comprends pas pourquoi l'on ne sort pas. Sylvie Catellin parle à propos de ce processus mental d'une « rupture qui génère un trouble dans l'entendement et qui exige une explication ${ }^{32}$ ». Cette explication viendra dans la fable par la découverte rétrospective par le lecteur-modèle de la véritable cause : la dévoration des animaux par le Lion. Catellin rapproche cette attitude de l'abduction telle que $\mathrm{C}$. S. Peirce la définit (toujours à propos de Zadig et valable chez La Fontaine) :

Elle consiste à partir d'un fait singulier ou surprenant, à inférer une proposition qui constitue l'hypothèse la plus plausible permettant d'expliquer ce fait. Elle infère, à partir de faits d'une sorte, des faits d'une autre sorte, alors que l'induction infère à partir d'un ensemble de faits à un autre ensemble de faits similaires ${ }^{33}$.

Catellin, poursuivant son exemple voltairien, approfondit les liens qui unissent «le raisonnement indiciaire » et la démarche scientifique :

Il [Voltaire] confère aussi une toute autre portée épistémologique au raisonnement indiciaire des princes au modèle cynégétique des chasseurs, selon l'expression de Carlo Guizburg. L'interprétation des indices fait ici appel à un outillage mental proche de la démarche scientifique, et qui servira de modèle au biologiste Thomas Huxley, en 1888, pour parler de la méthode des paléontologues ${ }^{34}$.

La Fontaine fut un disciple de Gassendi et l'ami intime de François Bernier. Ce lien entre logique et pensée scientifique dans les Fables, abondamment rappelé par J.-C. Darmon, ne s'en trouve que renforcé. 
Mais reprenons cette conclusion logique que la fable et son auteur invite à formuler : $S i$ on entre et qu'on ne sort pas, alors (c'est parce que) le Lion a mangé les Députés, soit $\left(p^{\wedge}-q\right)$ $\rightarrow r$. Reste à savoir si cette proposition est vraie en vérifiant ce qu'elle dénote. En nous référant à l'économie de la fable l'on obtient :

$-\mathrm{v}(p)$ = on entre, dénotant l'énoncé : « Les pas empreints sur la poussière ${ }^{35}$ ».

- Soit $\mathrm{v}(p)$ est vraie.

- $\mathrm{v}(\neg q)=$ on ne sort pas, dénotant l'énoncé : «Pas un[e empreinte de pas] ne marque de retour ${ }^{36} »$.

- Soit $\mathrm{v}(\neg q)$ est vraie.

- $\mathrm{v}(r)=$ le Lion a mangé les Députés. Une lecture attentive ne montre aucun énoncé susceptible de dénoter une telle proposition. Et l'on se rend compte que $(r)$ est bien la conclusion cachée: le lion mange les animaux que seule l'abduction et la «lumière naturelle » permettent de découvrir. Mais $(r)$ cependant reste d'une certaine manière une hypothèse à confirmer.

Dans cette fable, la vérification intervient, ou du moins est rendue possible par une autre prémisse : le roi-lion est «malade », qui une fois de plus n'a, avec notre énoncé initial, aucun rapport direct de causalité. Mais il prend toute sa valeur explicative dans l'abduction que la fable induit. Ainsi, la conclusion logique que le lecteur-modèle doit retenir est issu du raisonnement suivant :

- Le Lion est malade.

- [Donc il ne peut chasser par lui-même ou courir après ces proies].

- Il demande alors à ces proies de venir à lui.

- Ces proies entrent en sa tanière.

- Et ne ressortent pas.

- [Parce qu'il les a dévorés].

Sont mises entre crochets les prémisses manquantes qui permettent de conclure l'abduction/enthymème lafontainienne :

[L]'explication doit être une proposition qui conduise à la prédiction de faits observés comme conséquences nécessaires ou du moins très probables dans ces circonstances. Il faut alors adopter une hypothèse qui est vraisemblable en ellemême et qui rend les faits vraisemblables. L'étape au cours de laquelle on adopte une hypothèse en tant qu'elle est suggérée par les faits est ce que j'appelle abduction $^{37}$.

Bien sûr l'énoncé implicite Parce qu'il les a dévorés est à mettre sur le compte de la nature même du pouvoir absolutiste que La Fontaine suggère plus qu'il ne décrit. Cette ultime conclusion de la fable devenant « ce qu'il fallait démontrer » autant que ce que «prouve » le corps de la fable. D'autre part et comme J.-C. Darmon l'a déjà évoqué, il resterait encore bien des mécanismes à décrire, en particulier l'administration de la preuve dans la fable. Ainsi, et juste à titre d'exemple, comment mettre en cause le Lion dans la dévoration des Députés? N’y aurait-il pas d'autres explications possibles: les animaux se sont suicidés, il y a une sortie de secours secrète, etc. C'est là nous éloigner quelque peu de notre objectif initial, mais c'est aussi montrer la nature même de l'abduction :

Au départ, le degré de probabilité de l'abduction reste faible. On ne peut pas affirmer avec certitude qu'une explication constitue la cause réelle d'une observation. L'incertitude peut porter aussi bien sur la plausibilité de l'explication que sur la validité de la connaissance permettant l'explication ${ }^{38}$. 
31 Ici, ces règles logiques sont encore très largement implicites, mais elles ont, comme le suggère plus qu'il ne l'explique J.-C. Darmon chez La Fontaine, un lien très étroit avec l'enthymème et la «méthode d'invention » dans sa version gassendiste, via François Bernier :

Par ce type d'effet, certaines fables défient l'esprit et l'imagination du lecteur, en mettant en spectacle et en énigme l'inférence elle-même, en tant qu'action, choix entre plusieurs lignes de sens possibles ${ }^{39}$.

Ces règles ne sont « déductibles » qu'à partir de la « lumière naturelle » dont on serait bien en peine d'expliciter le fonctionnement. Pourtant, l'abduction lafontainienne, bien que fabuleuse n'en est pas pour autant délirante ou irrationnelle, comme J.-C. Darmon le remarque pour d'autres fables qu'il a analysées :

Une logique, même, qui, de façon très symptomatique, lorsqu'il s'agit d'exposer la "méthode d'invention", suggère une part d'indétermination et d'aléas dans l'invention même du syllogisme. Tout lecteur de La Fontaine se sentirait sans doute, une fois de plus, en terres familières en voyant surgir, au sein d'un des derniers chapitres de cette logique simple mais austère, la figure du flair animal pour métaphoriser ce qu'est, en tout syllogisme, la quête du Moyen ${ }^{40}$.

Comme le rappelle Philippe Hamou, « la quête du Moyen » gassendiste correspond à :

la « méthode d'invention » [qui] apprend plus particulièrement à trouver le moyen terme dans un raisonnement, à savoir ce qui permet de passer des prémisses à la conclusion, soit par voie de composition (synthèse), soit par voie de résolution (analyse) ${ }^{41}$.

34 Prises en ce sens gassendiste, les prémisses manquantes de La Fontaine, le Lion est malade, Donc il ne peut chasser par lui-même ou courir après ces proies, correspondent assez bien à cette quête du Moyen terme que l'esprit parvient sans mal à "suppléer ». Ici la solution est d'abord obtenue par une forme d'inhérence pragmatique : un lion malade ne peut pas courir. Là, le Lion a dévoré les Députés, s'obtient par une forme d'analyse induite par le reste des prémisses :

Il convient de remarquer que le mot même de «méthode d'invention » utilisé ici sans hésitation pour désigner l'art de découvrir les propositions moyennes aurait $\mathrm{pu}$ passer anciennement pour un oxymore, en un temps où l'invention et la méthode constituaient deux étapes logiquement distinctes et nécessairement successives. Gassendi l'emploie sans sourciller et mobilise pour le penser des ressources conceptuelles qui ne sont plus celle de la rhétorique, mais celle des mathématiques. C'est la manière de démontrer des géomètres, par analyse (resolutio), c'est-à-dire en remontant des conséquences aux principes, et par synthèse (compositio) en procédant des principes aux conséquences, qui lui sert de modèle pour penser cette nouvelle logique de la découverte ${ }^{42}$.

\section{Conclusion}

Cette analyse de la fable lafontainienne met en scène et donne à voir l'analyse ellemême, ou comme le dit bien mieux Darmon: "Par ce type d'effet, certaines fables défient l'esprit et l'imagination du lecteur, en mettant en spectacle et en énigme l'inférence elle-même, en tant qu'action, choix entre plusieurs lignes de sens possibles ${ }^{43}$. » La « méthode d'invention » fait partie de l'arsenal rhétorique utilisé par le fabuliste pour parvenir au niveau réflexif (épistémologique) du discours philosophique. Mais plus que la philosophie de la connaissance de La Fontaine, nous avons souhaité montrer par où, une même "méthode ", une même procédure «logique » (qui relève 
de fait de la psychologie de l'intelligence) portée par le " paradigme indiciaire » pouvait lier discours fabuleux et pensée savante. Il nous resterait aussi à nous interroger, chez La Fontaine, sur l'articulation de l'abduction et de la prudence.

\section{NOTES}

1. J.-C. Darmon, Philosophies de la Fable. Poésie et pensée dans l'œuvre de La Fontaine, Paris, éditions Hermann, 2011, p. 13.

2. S. Rahman (avec la collaboration de L. Keiff), «Dialogique standard : notions fondamentales », http://stl.recherche.univ-lille3.fr/sitespersonnels/rahman/rahmancours1.html [consulté le 15/12/2015].

3. O. Caïra, Définir la fiction, Paris, EHESS, 2011, p. 36.

4. J. Lassègue, Turing, Les Belles-Lettres, 1988, cité dans O. Caïra, op. cit., p. 36.

5. J.-P. Sermain, Métafictions (1670-1730). La réflexivité dans la littérature d'imagination, Paris, Honoré Champion, 2002, p. 436-437. Le concept de métafiction peut se décrire comme le fait pour une fiction de prendre " pour objet une fiction et [de] se constitue[r] comme réflexion à son égard ».

6. J. de La Fontaine, «Les Animaux malades de la Peste », VII, 1, dans Fables, éd. J.-C. Darmon, Paris, Librairie Générale Française, 2002.

7. A. Arnaud et P. Nicole, La Logique ou l'art de penser, éd. D. Descote, Paris, Honoré Champion, 2014, p. 389-390. Je souligne.

8. J. de La Fontaine, «Préface », op. cit., p. 41. Je souligne.

9. J. de La Fontaine, « Le Lion malade et le renard », VI, 14, op. cit.

10. J. J. Vega y Vega, L'enthymème: Histoire et actualité de l'inférence du discours, Lyon, PUL, 2000, p. 40. Les italiques sont de l'auteur.

11. Vv. 23-24.

12. A. Arnauld et P. Nicole, op. cit. p. 241.

13. L. Vax, «Barbara, Celarent, Darii, Ferio, Baralipton », Le Portique [En ligne].

14. B. Walliser, D. Zwirn et H. Zwirn, «Raisonnements non certains et changement de croyances ", dans T. Martin éd., Probabilités subjectives et rationalité de l'action, Paris, CNRS éditions, 2003, p. 47-66.

15. N. Clerbout, «Logique propositionnelle »,Vade-mecum de l'étudiant de Licence 1 ; http:// stl.recherche.univ-lille3.fr/sitespersonnels/rahman/rahmancours1.html [consulté le 21/03/2014].

16. Au vers 15

17. Au vers 18 .

18. N. Clerbout, op. cit.

19. A. Arnauld et P. Nicole, op. cit., p. 123.

20. Vv.13-19.

21. Voir A. Arnauld et P. Nicole, op. cit., III, 19, p. 467-468.

22. Marin Cureau de La Chambre, L'Art de connaître les hommes, Amsterdam, le Jeune, 1660, p. 274.

23. A. Arnauld et P. Nicole, op. cit., p. 679.

24. J. de La Fontaine, « Les Frelons et les mouches à miel », I, 21, dans Fables, op. cit., p. 83-85.

25. Nous mobilisons, par simplification, la théorie aristotélicienne du signe, en éludant volontairement la question historique de celle(s) utilisée(s) par La Fontaine. 
26. S. Catellin, Sérendipité, Du conte au concept, Paris, Seuil, 2014. p. 49. Tous les mots en italique sont de l'auteur.

27. Le Voyage et les avantures des trois princes de Sarendip publié en 1719.

28. S. Catellin, op. cit., p. 73.

29. $S$. Catellin montrera par la suite que cette définition rudimentaire et naïve est très insuffisante.

30. Ibid. p. 179.

31. V.19.

32. S. Catellin, op. cit., p. 179.

33. Ibid., p. 71.

34. Ibid., p. 49.

35. V.15.

36. V.18.

37. C.S. Peirce, Collected Papers, 8 vol, éd. (1-6) C. Hartshorne \& P. Weiss et (7-8) éd. A. Burks, Cambridge, Harvard University Press, 1931-1958, vol.7, p. 202; cité dans C. Chauviré, "Aux sources de la théorie de l'enquête : la logique de l'abduction en Peirce ", Revista Colombiana de Filosofía de la Ciencia, vol. X, núm. 20-21, 2010, p. 27-56, Universidad El Bosque Colombia.

38. S. Catellin, op. cit., p. 75.

39. J.-C. Darmon, Philosophies de la Fable, op. cit., p. 275. Je souligne.

40. Ibid., p. 273.

41. P. Hamou, «Sur les origines du concept de méthode à l'âge classique : La Ramée, Bacon et Descartes ", Revue LISA/LISA e-journal. [En ligne], vol. XII-n5|2014, mis en ligne le 14 octobre 2014, consulté le 27 octobre 2015. URL : http://lisa.revues.org/6249 ; DOI : 10.4000/lisa.6249.

42. Ibid.

43. J.-C. Darmon, Philosophies de la Fable, op. cit., p. 275. Je souligne.

\section{AUTEUR}

FRANCK BARON

EHESS / GRIHL 